\title{
Analysis of Various Edge Detection Techniques
}

\author{
Anuradha Karnam, Deepti R. Kulkarni, Kshama P. Sunagar, \\ Nikhita G. Revankar and Mahendra M. Dixit
}

\begin{abstract}
In this paper the fundamental concept of various edge detection techniques are studied. It mainly concentrates on gradient based Robert, Sobel, Prewitt and Canny edge detection operators. They are implemented using MATLAB and Simulink.
\end{abstract}

Keywords--- Gradient, Canny, Sobel, Robert, Prewitt.

\section{INTRODUCTION}

O NE of the fundamental problem in the area of image processing is the location of boundaries of objects within an image. A considerable number of edge detection schemes have been devised. The comparison is necessary in order to compare the various available techniques. It establishes performance limitations of each scheme under the various threshold values. The edge detection techniques studied in this paper are Sobel, Roberts, Prewitt and Canny edge detection. The figure 1 shows block diagram of different types of edge detection.

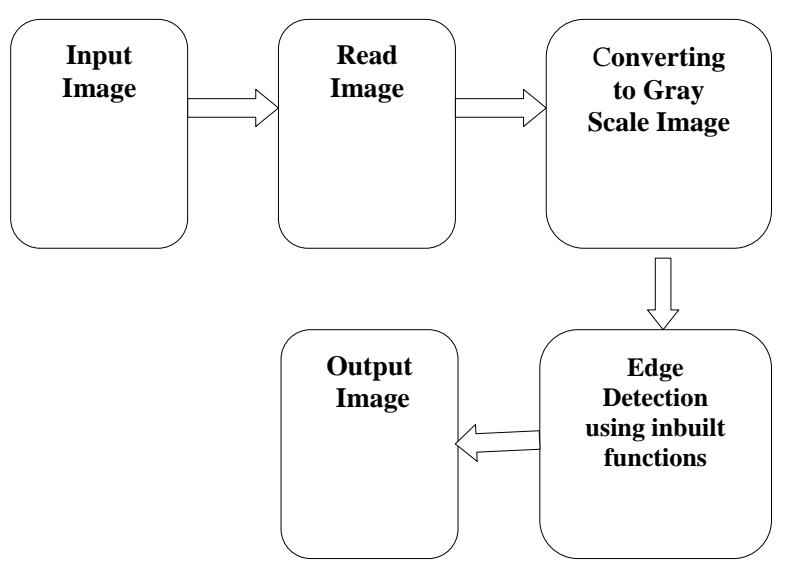

Figure 1: Block Diagram for Different Types of Edge Detection

\section{TECHNiques UsEd for Edge Detection}

The different edge detection techniques studied are

1. Sobel Operator

Anuradha Karnam, Department of Electronics and Communication Engineering, SDMCET, India. E-mail:anuradhakarnam299@gmail.com

Deepti R. Kulkarni, Department of Electronics and Communication Engineering, SDMCET, India.E-mail:deepti_14rk@yahoo.com

Kshama P. Sunagar, Department of Electronics and Communication Engineering, SDMCET, India. E-mail:kshamaps320@gmail.com

Nikhita G. Revankar, Department of Electronics and Communication Engineering, SDMCET, India.E-mail:nikitarevankar06@gmail.com

Mahendra M. Dixit, Department of Electronics and Communication Engineering, SDMCET, India. E-mail:mmdixitmm@yahoo.co.in

DOI: 10.9756/BIJRCE.8190
2. Prewitt Operator

3. Robert's Cross Operator

4. Canny Operator.

\section{A. Sobel Operator}

It has a pair of $3 \times 3$ convolution kernels as shown in table1. Other kernel is rotated by $90^{\circ}$.

Table 1: Masks used by Sobel Operator

\begin{tabular}{|l|l|l|}
\hline-1 & 0 & 1 \\
\hline-2 & 0 & 2 \\
\hline-1 & 0 & 1 \\
\hline
\end{tabular}

Gx

\begin{tabular}{|c|c|c|}
\hline 1 & 2 & 1 \\
\hline 0 & 0 & 0 \\
\hline-1 & -2 & -1 \\
\hline
\end{tabular}

Gy
The kernels can be applied separately to the input image, to produce separate measurements of the gradient component in each orientation ( $\mathrm{Gx}$ and $\mathrm{Gy}$ ). The gradient magnitude is given by:

$$
|G|=\sqrt{ }\left(\mathrm{Gx}^{2}+\mathrm{Gy}^{2}\right)
$$

An approximate magnitude is computed using:

$$
|G|=|G x|+|G y|
$$

The angle of orientation of the edge is given by:

$$
\theta=\arctan (\mathrm{Gy} / \mathrm{Gx})
$$

\section{B. Prewitt Operator}

Prewitt operator is similar to the Sobel operator and is used for detecting vertical and horizontal edges in images. It uses the same equations as the Sobel operator. It has $3 \times 3$ convolution kernels as shown in table 2 .

Table 2: Masks used by Prewitt Operator

\begin{tabular}{|c|l|l|}
\hline-1 & 0 & 1 \\
\hline-1 & 0 & 1 \\
\hline-1 & 0 & 1 \\
\hline
\end{tabular}

$\mathrm{Gx}$

\begin{tabular}{|c|c|c|}
\hline 1 & 1 & 1 \\
\hline 0 & 0 & 0 \\
\hline-1 & -1 & -1 \\
\hline
\end{tabular}

Gy
It does not place any emphasis on pixels that are closer to the center of the masks.

\section{Robert's Cross Operator}

The Roberts Cross operator performs a 2-D spatial gradient measurement on an image. This operator has a pair of $2 \times 2$ convolution kernels as shown in table.3. Other kernel is rotated by $90^{\circ}$ [3]. 
Table 3: Masks used by Robert's Operator

\begin{tabular}{|l|l|}
\hline+1 & 0 \\
\hline 0 & -1 \\
\hline
\end{tabular}

Gx

\begin{tabular}{|l|l|}
\hline 0 & +1 \\
\hline-1 & 0 \\
\hline
\end{tabular}

Gy
The kerneis are appıed to ine input image, to produce the gradient component Gx and Gy. The gradient magnitude is given by:

$$
|G|=\sqrt{ }\left(G x^{2}+G y^{2}\right)
$$

an approximate magnitude is computed using:

$$
|G|=|G x|+|G y|
$$

The angle of orientation of the edge is given by:

$$
\theta=\arctan (G y / G x)-3 \pi / 4
$$

\section{Canny Operator}

Steps involved in canny edge detection algorithm are as follows:

1. First it smoothes the image to eliminate the noise.

2. It then finds the image gradient to highlight regions with high spatial derivatives.

3. The present pixel value is compared with neighbouring pixel values. If pixel value is greater then it masks in the same direction else the value is suppressed (non-maximum suppression).

4. High threshold and low threshold values are fixed. If the pixel value is greater than the high threshold value then it is a strong edge. If the value falls in-between the double threshold values then it is a weak edge. Pixel is discarded if its value is less than low threshold value.

5. Edges are tracked by using hysteresis.

\section{RESULTS}

The above four technique were implemented in MATLAB and Simulink ${ }^{\circledR}$ and the results are shown in figure 3. Qualitative analysis and subjective analysis were made on the obtained results. Table 4 shows comparison canny edge detector in Matlab and Simulink. The threshold was varied between Threshold $=[0.01-0.09]$ to Threshold $=[0.01-0.4]$. Table 5 shows the comparison of various threshold values of four edge detectors.

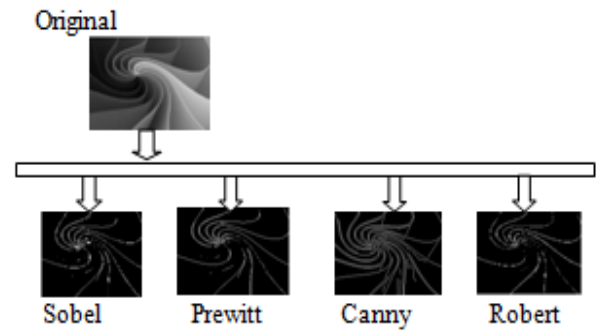

Table 4: Comparison of Matlab and Simulink Results for Canny Edge Detector

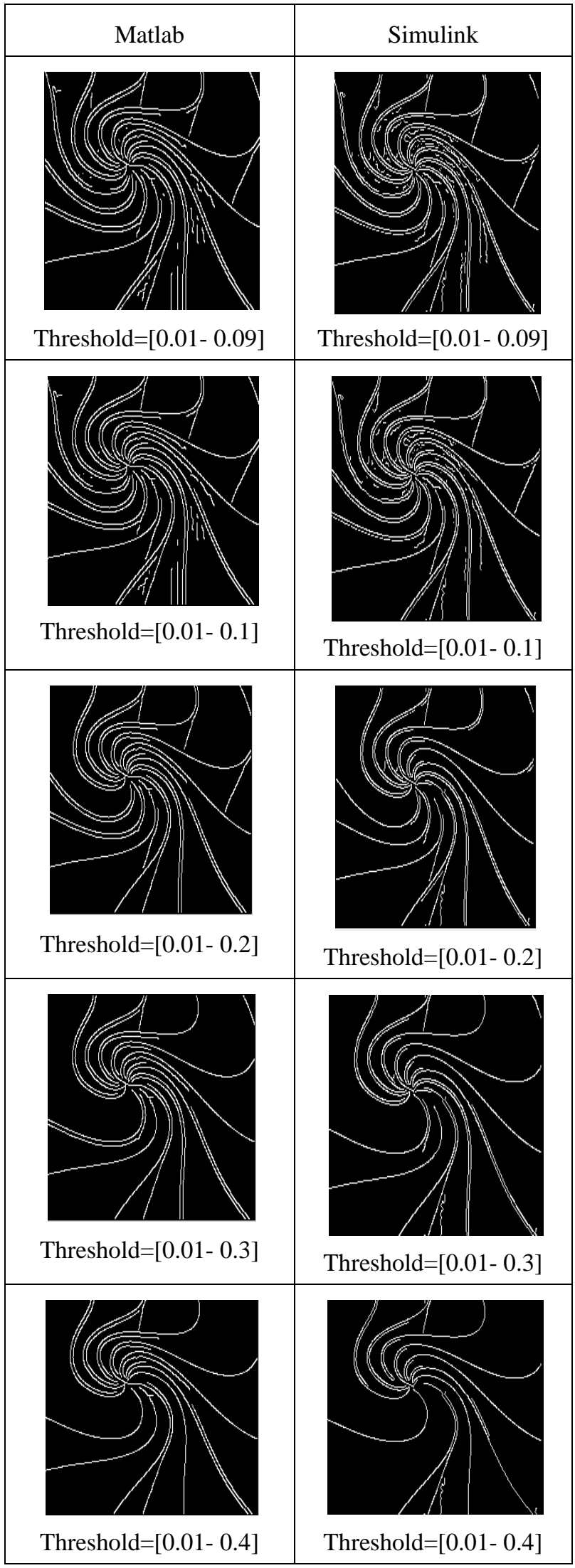

Figure 2: Simulink Results 
Table 5: Comparison of Various Threshold Values of Four Edge Detectors

\begin{tabular}{|c|c|c|c|}
\hline Sobel & Prewiit & Roberts & Canny \\
\hline Threshold $=0.1$ & Threshold $=0.1$ & Threshold $=0.1$ & $\begin{array}{c}\mathrm{Th}=[0.01- \\
0.09]\end{array}$ \\
\hline Threshold $=0.5$ & Threshold $=0.5$ & Threshold $=0.5$ & $\begin{array}{c}\mathrm{Th}=[0.01- \\
0.3]\end{array}$ \\
\hline Threshold=2 & Threshold=2 & Threshold=2 & $\begin{array}{c}\text { Th }=[0.01- \\
0.3]\end{array}$ \\
\hline Threshold=4 & Threshold $=4$ & Threshold=4 & $\begin{array}{c}\text { Th }=[0.01- \\
0.4]\end{array}$ \\
\hline Threshold=12 & Threshold=12 & Threshold $=12$ & $\begin{array}{c}\mathrm{Th}=[0.01- \\
0.5]\end{array}$ \\
\hline
\end{tabular}

\section{CONCLUSION}

Canny edge detection method is the efficient technique amongst all the available algorithms because of its noise suppression and clear edge detection.

\section{FUTURE SCOPE}

In the future, edge detection can be implemented in low cost hardware such as Beagle bone raspberry-pi and arduino. This can be extended to other industrial applications.

\section{REFERENCES}

[1] https://wikipedia.org/wiki/Matlab.

[2] R. Maini and H. Aggarwal, "Study and comparison of various image edge detection techniques", International journal of image processing (IJIP), Vol.3, No.1, Pp.1-11, 2009.

[3] J.R. Fram and E.S. Deutsch, "On the quantitative evaluation of edge detection schemes and their comparison with human performance", IEEE Transactions on Computers, Vol.100, No.6, Pp.616-628, 1975.

[4] S.K. Katiyar and P.V. Arun, "Comparative analysis of common edge detection techniques in context of object extraction", IEEE IGRS, Vol.50, No.11b, 2014.

[5] G.T. Shrivakshan and C. Chandrasekar, "A comparison of various edge detection techniques used in image processing", IJCSI International Journal of Computer Science Issues, Vol.9, No.5, Pp.272-276, 2012.

[6] Y.K. Singh, "Multi-level edge detectors based on the convolution matrices of base lengths 2 and 3", ARPN Journal of Engineering and Applied Sciences, Vol.6, No.1, Pp.29-37, 2011.
[7] O.R. Vincent and O. Folorunso, "A descriptive algorithm for sobel image edge detection", Proceedings of Informing Science\&IT Education Conference (InSITE), Vol.40, Pp.97-107, 2009.

[8] E. Aybar, "Sobel edge detection method for matlab", Anadolu University, Porsuk Vocational School, 26410, 2006.

[9] T.H.H. Lee and T.R. Taipei, "Edge detection analysis", Graduate Institute of Communication Engineering, National Taiwan University, Taipei, Taiwan, ROC, 2007. 